TRENDS IN HYDROZOAN BIOLOGY - IV. C.E. MILLS, F. BOERO, A. MIGOTTO and J.M. GILI (eds.)

\title{
Structure of an epiphytic hydroid community on Cystoseira at two sites of different wave exposure*
}

\author{
ANUSCHKA FAUCCI ${ }^{1}$ and FERDINANDO BOERO ${ }^{2}$ \\ ${ }^{1}$ Kewalo Marine Laboratory, University of Hawaii, Honolulu, USA. E-mail: anuschka@ hawaii.edu \\ ${ }^{2}$ Dipartimento di Biologia, Università di Lecce, 73100 Lecce, Italy. E-mail: boero@unile.it
}

\begin{abstract}
SUMMARY: Epiphytism is a strategy by which opportunistic species such as hydroids, escape the intense levels of competition in marine hard bottom communities. Species of the macroalgae Cystoseira have a seasonal turnover of the frond, and we hypothesise that epiphytic hydroids colonising such an unstable substrate might show some degree of specialisation. Here the first systematic study on hydroid-Cystoseira communities is presented. In particular, the seasonal and spatial distribution of epiphytic hydroids on three species of Cystoseira at two sites of different wave exposure at Porto Cesareo (Ionian Sea/Italy) were investigated. Thirty-two hydroid species were recorded which are well known from other substrates and thus are not specific to Cystoseira; dominant species were all thecates. The influence of biological factors such as competition and the structure and abundance of the host, seem to have little influence on the hydroid community. The factors of greatest influence were mostly abiotic: sedimentation rate, nutrient levels, temperature and most especially water movement. The importance of water movement was evident in the higher colonisation of algal stems, higher hydroid frequency, larger colonies, reduced colony height, species composition, and distribution on the stems at the wave-exposed site.
\end{abstract}

Key words: epiphytic hydroids, water movement, Cystoseira, Mediterranean.

\section{INTRODUCTION}

The environmental factors limiting species distribution in marine benthic communities are temperature, light, water movement and substrate availability, among others. Temperature depends on water currents, depth and season, and influences nutrition, growth, life cycles and reproduction. Water movement supplies the organisms with food, nutrients and dissolved gases, prevents accumulation of sediments, and disperses waste products and larvae, but also acts as a mechanical stress factor. Many organisms have evolved morphological adaptations to withstand such stress. These structural responses are

*Received February 17, 1999. Accepted May 15, 2000. reflected by the different distribution of families, species, or morpho-types due to differences in water movement intensity (Riedl, 1966). Among sessile organisms increasing intensity of water movement induces smaller size, lower colony height, higher population density, and a morphology that withstands high mechanical stress (Riedl, 1971; Boero, 1981b, 1984). The distribution of adults is also the result of competition and settlement behaviour, which is regulated by larval substrate choice (Hayward, 1980).

To avoid competition for space a variety of strategies have evolved. One of the most common of these strategies is epiphytism, which reduces competition because fewer species are encountered and continuous growth of the host produces new substrate for 
less competitive opportunistic species (Seed, 1985). However, compared to living directly on rock, using plants as a substrate limits survival because of the turnover of host tissue and higher mechanical stress due to water movement and the flexibility of the substrate. Therefore an epiphytic life style requires specific adaptations (Seed, 1985), like fast growth to a small body size, early sexual or asexual reproduction, and a short life span (Coma et al., 1992).

Hydroids are among the first colonisers of newly exposed hard substrates and often compete poorly with other sessile organisms (Gili and Hughes, 1995). Most hydroids are substrate generalists, but several species grow on a limited range of surface types. They are especially abundant on seaweeds and their distribution on these hosts is due to different preferences for various aspects of the physical environment, notably water movement and sediment deposition, and substrate choice by larvae, both between and within individual plants (Seed, 1985). The choice appears to be related to both the chemical nature and the structure and texture of the plant surface (Katô et al., 1975; Seed, 1985). Compared to other sessile invertebrates, hydroids grow fast and have a short life span. Together with their, mostly, asexual method of reproduction, these characteristics make them successful epiphytes.

The different hydroid species differ in several features, like reproduction, dispersal and morphology. Thecate hydroids are able to withstand higher mechanical stress than athecates, because of their protective theca (Riedl, 1966). All hydroids have a mainly sessile polyp stage in their life cycle, but many species have a life cycle involving a free-living medusa stage, which can live for several months in the plankton before reproducing sexually. Although reproductive strategy does not seem to influence greatly the colonisation capabilities of a species (Boero, 1984), in epiphytic communities, suppression of the medusa stage and reduction in the frequency of sexual reproduction is common (Boero, 1987).

Among previous studies on epiphytic hydroids the most substantial are those on Posidonia oceanica (Boero, 1981a; Garcia Rubies, 1987), Halimeda tuna (Llobet et al., 1991), Amphibolis (Watson, 1992), Sargassum (Nishihira, 1966), Fucus serratus (Seed et al., 1981) and Laminaria (Schultze et al., 1990). Most of these investigations have been carried out on hydroids that settle on seagrasses with two-dimensional leaves or algae with laminar fronds, which facilitate the quantification and study of the different epiphytes.
Dendroid seaweeds of the genus Cystoseira (Fucales, Phaeophyceae) are very abundant in the Mediterranean Sea, growing from the surface down to $100 \mathrm{~m}$ depth. Because species distribution depends on wave-exposure, depth and water quality, they are good descriptors of the zonation of the photophilous vegetation on hard bottom substrates (Amico et al., 1985). Species of Cystoseira also differ in their morphology, surface characteristics of their stems and in their chemistry (Amico, 1995). As they belong to the hemiphanerophycean brown algae, they have a seasonal turnover of tissue. Their phylloids and branches of higher order are shed in the unfavourable season, usually winter, while the holdfast and primary branches persist (Cormaci, 1995). Therefore this substrate is not always available for epiphytes during the year. Epiphytic algae and other organisms on Cystoseira have been studied by Arrontes (1990), Otero-Schmitt and PérezCirera (1996) and Russo (1997), while previous studies of hydroids on Cystoseira (e.g. Riedl, 1966) provide only scattered observations.

Here, the first systematic investigation of epiphytic hydroids on Cystoseira is presented. In particular, the seasonal and spatial distribution of epiphytic hydroids on three species, Cystoseira amentacea Bory, C. barbata (Goodenough et Woodward) and $C$. compressa (Esper), at two sites of different wave exposure on the rocky shore of Porto Cesareo (Ionian Sea/Italy) was studied. The following questions were addressed: 1) what are the characteristics of the hydroid community growing on Cystoseira? 2) Is there seasonality? 3) Does exposure to wave action influence the Cystoseira-hydroid community structure? 4) Do the characteristics of the algal species influence the hydroid communities? 5) Do differences in morphology, such as the presence or absence of a theca influence the distribution of hydroid species epiphytic on Cystoseira?

\section{METHODS}

The study was carried out on the rocky shore of the Ionian Sea at Porto Cesareo/Italy between July 1996 and July 1997. Algal stems were collected by SCUBA at $0.5,2$ and $3 \mathrm{~m}$ depth along four transects at two sites of different wave-exposure. For depth and transect, 5 stems of each of the three species of Cystoseira (C. barbata, C. compressa, C. amentacea) were collected within an area of $50 \times 50 \mathrm{~cm}$. Samples were taken monthly, weather conditions 
permitting, and on each sampling date water temperature was measured. Samples were examined under a binocular.

The following data were taken for each algal stem: a) maximal stem length $(\mathrm{cm})$, as a measure for the amount of substrate available and the seasonality of the algae; b) the hydroid species present; c) estimated hydroid colony size, i.e. abundance of the hydroid colonies on the stem (1: present, 2: abundant, 3: very abundant (Boero and Fresi, 1986)) where all polyps of the same species on the stem were assumed to belong to the same colony; d) distribution of the hydroid colonies on the stem (1: stipe and holdfast, 2: inner or intermediate part of the stem, 3: outer part of the stem (Arrontes, 1990)). The following diversity indices were calculated for the hydroid communities epiphytic on the stems of the three species of Cystoseira and from the two sites. Community diversity was measured by species number, Shannon-Wiener index of diversity $\left(H^{\prime}\right)$,

$$
\mathrm{H}^{\prime}=-\sum_{\mathrm{i}=1}^{\mathrm{s}} \mathrm{p}_{\mathrm{i}} \ln \mathrm{p}_{\mathrm{i}},
$$

where $S=$ number of species and $p_{i}=$ proportion of total sample belonging to ith species of total algal stems, and Pielou's index of evenness $(\mathrm{J}), \mathrm{J}=\mathrm{H}^{\prime} /$ ln $\mathrm{S}$, where $\mathrm{H}^{\prime}=$ Shannon-Wiener index and $\mathrm{S}=$ number of species (Krebs, 1989). These two indices were tested for significance by a randomisation test (Solow, 1993). Species dominance was measured by Simpson's dominance index $\left(\mathrm{I}_{\mathrm{dom}}\right)$ (Watson, 1992).

Ordination by Canonical Correspondence Analysis (CCA) (Ter Braak, 1986) was used to summarise the variation in species composition and frequency related to site, including their environmental variables, and season. The environmental variables were water depth $(\mathrm{m})$, water temperature $\left({ }^{\circ} \mathrm{C}\right)$, algal stem length $(\mathrm{cm})$, number of algal stems collected, percentage of algal stems colonised, degree of algal structure (1: low structured, smooth surface; 2 : high structured, smooth surface; 3: high structured, rugose surface), and dummy variables for exposed/sheltered and the three species of Cystoseira. As a measure of goodness of the ordination, species-environment correlations for the first two ordination axes were used. Significance levels of the ordinations were computed by a Monte Carlo permutation test. Ordination analysis and Monte Carlo permutation test were computed using the CANOCO program package developed by Ter Braak (1988).

\section{RESULTS}

Thirty-two hydroid species, twelve athecate and twenty thecate species, were recorded on the three species of Cystoseira (Table 1). Nine species have a free-living medusa, three have liberable eumedusoids, and twenty have fixed gonophores.

\section{Hydroid abundance and distribution}

The number of hydroid species varied from zero to seven per algal stem. Most stems from the waveexposed site were colonised by one to three species, whereas stems from the sheltered site were mostly colonised by zero or one species. With a maximum of four species per stem, C. compressa was colonised by fewer species per stem than the other species of Cystoseira, both with maximal seven species per stem.

The percentage of stems colonised by at least one hydroid ranged during the year from $15 \%$ to $100 \%$ and was typically higher at the exposed site than at the sheltered one (Fig. 1). Colonisation was highest in autumn, but declined in winter/spring at the sheltered site, especially on $C$. compressa. This decline in colonisation correlates with the decline of algal stem length. C. compressa was on average less colonised than $C$. barbata and $C$. amentacea.

The abundance of the hydroid colonies on a single algal stem (Table 2a) belonged mostly to the categories 1 (present) and 2 (abundant). The difference of colony-abundance on the three species of Cystoseira was significant between the hydroids from the exposed site (Chi-square Test: $\mathrm{p}=0.004)$ and the thecates from both sites $(p=0.008, p=0.003)$, but not between hydroids from the sheltered site or between athecates (p-value between 0.05 and 0.16). The differences between sites were always significant (Chisquare Test: $\mathrm{p}<0.0001)$. At the wave-exposed site more colonies belonged to category 3 (very abundant) than at the sheltered site.

The general distribution of the hydroids on the algal stem is shown in Table $2 \mathrm{~b}$. The central parts (category 2) of the algal stems were generally the most evenly colonised ones. The outer parts (category 3) showed the lowest colonisation, especially at the wave-exposed site. At the sheltered site the athecate hydroids were primarily distributed between the central parts of the stem and its base, whereas the thecate species were more often distributed on the central and outer parts. The differences between the sites and athecates/thecates were significant (Chi-square Test: $\mathrm{p}<0.0001$ ). 
TABLE 1. - Hydroid species recorded on Cystoseira. Specimens of Aglaophenia indet. were not identifed at species level mostly because of their small size; specimens of Clytia sp. belong either to Clytia hemisphaerica or perhaps a new species of Clytia. x: present, f: fertile, p: asexual propagules; g: fixed gonophores, m: medusae, mg: liberable eumedusoids or swimming gonophores; E: endemic, MA: Mediterranean Atlantic, B: boreal, TA: tropical Atlantic, IP: Indo-Pacific, CT: circumtropical, C: cosmopolitan, nc: non classifiable. (after Boero and Bouillon, 1993)

$\mathrm{N}^{\mathrm{o}}$ species

exposed site

sheltered site

\begin{tabular}{|c|c|c|c|c|c|c|c|c|c|c|c|c|c|c|c|c|c|c|c|}
\hline & & $\begin{array}{l}\frac{0}{0} \\
\text { J } \\
\stackrel{\Xi}{\Xi}\end{array}$ & 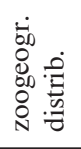 & 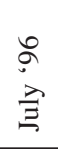 & 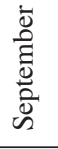 & $\begin{array}{l}\overline{0} \\
0 \\
0 \\
0\end{array}$ & $\begin{array}{l}\vec{E} \\
\overrightarrow{0} \\
0 \\
0 \\
0\end{array}$ & $\overline{\bar{z}}$ & $\stackrel{\Xi}{\Xi}$ & $\stackrel{\lambda}{\Xi}$ & & 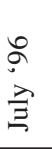 & 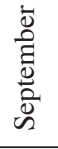 & 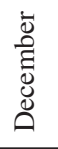 & $\begin{array}{l}\vec{\Xi} \\
\stackrel{\Xi}{\Xi} \\
\text { ज्ञ }\end{array}$ & 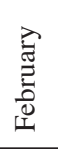 & $\overline{\vec{Z}}$ & $\sum_{\Sigma}^{\vec{J}}$ & \\
\hline 1 & Clava multicornis (Forskal, 1775) & $\mathrm{g}$ & B & & & & $\mathrm{x}$ & & & & & & & $\mathrm{x}$ & $\mathrm{x}$ & $\mathrm{x}$ & & & \\
\hline 2 & Eudendrium glomeratum Picard, 1952 & $\mathrm{~g}$ & CT? & & & & & & & & & & & & & $\mathrm{x}$ & $\mathrm{x}$ & & \\
\hline 3 & Eudendrium motzkossowskae Picard, 1952 & $\mathrm{~g}$ & TA & & $\mathrm{x}$ & $\mathrm{x}$ & $\mathrm{x}$ & $\mathrm{x}$ & $\mathrm{x}$ & & & $\mathrm{x}$ & $\mathrm{x}$ & $\mathrm{x}$ & $\mathrm{x}$ & $\mathrm{x}$ & $\mathrm{x}$ & $\mathrm{x}$ & \\
\hline 4 & Eudendrium ramosum (L., 1758) & $\mathrm{g}$ & $\mathrm{C}$ & & & & & & & & & & & $\mathrm{x}$ & & & & & \\
\hline 5 & Hydractinia fucicola (M. Sars, 1857) & $\mathrm{g}$ & MA & & $\mathrm{x}$ & $\mathrm{x}$ & $\mathrm{x}$ & $\mathrm{x}$ & $\mathrm{x}$ & & & & $\mathrm{x}$ & $\mathrm{x}$ & & $\mathrm{x}$ & $\mathrm{x}$ & $\mathrm{f}$ & \\
\hline 6 & Cladonema radiatum Dujardin, 1843 & $\mathrm{~m}$ & $\mathrm{CT}$ & & & & & & & & & & & & & $\mathrm{x}$ & & & \\
\hline 7 & Coryne muscoides $(\mathrm{L} ., 1761)$ & $\mathrm{g}$ & MA & & $\mathrm{f}$ & $\mathrm{f}$ & $\mathrm{x}$ & $\mathrm{f}$ & & & & $\mathrm{x}$ & $\mathrm{f}$ & $\mathrm{x}$ & $\mathrm{x}$ & $\mathrm{f}$ & $\mathrm{x}$ & $\mathrm{x}$ & \\
\hline 8 & Coryne pusilla Gaertner, 1774 & $\mathrm{~g}$ & B & & & & & & & & & $\mathrm{x}$ & & & & $\mathrm{x}$ & & & \\
\hline 9 & Sarsia producta (Wright, 1858) & $\mathrm{m}$ & TA & & & & $\mathrm{x}$ & & & & & & & & & & & & \\
\hline 10 & Ectopleura wrighti Petersen, 1979 & $\mathrm{~m}$ & $\mathrm{E}$ & & & & & & & & & & & $\mathrm{x}$ & & & $\mathrm{x}$ & & \\
\hline 11 & Cladocoryne floccosa Rotch, 1871 & $\mathrm{~g}$ & CT & & $\mathrm{x}$ & & & & & & & & $\mathrm{x}$ & $\mathrm{x}$ & $\mathrm{x}$ & $\mathrm{x}$ & $\mathrm{x}$ & $\mathrm{x}$ & \\
\hline 12 & Zanclea sp. see Gravili et al., 1996 & $\mathrm{~m}$ & & & & & $\mathrm{x}$ & & & & & & & $\mathrm{x}$ & $\mathrm{x}$ & $\mathrm{x}$ & & & \\
\hline 13 & Eirene viridula (Péron and Lesueur, 1810) & $\mathrm{m}$ & IP & & & & $\mathrm{x}$ & $\mathrm{x}$ & & & & & & & & & $\mathrm{x}$ & & \\
\hline 14 & Mitrocoma annae Haeckel, 1864 & $\mathrm{~m}$ & $\mathrm{E}$ & & & & & & & & & & & & & $\mathrm{x}$ & & & \\
\hline 15 & Anthohebella parasitica (Ciamician, 1880) & $\mathrm{mg}$ & $\mathrm{nc}$ & & & & $\mathrm{x}$ & $\mathrm{x}$ & & & & & & & & & & & \\
\hline 16 & Halecium nanum Alder, 1859 & $\mathrm{~g}$ & TA & & & & $\mathrm{x}$ & $\mathrm{x}$ & $\mathrm{x}$ & & & & & & $\mathrm{x}$ & $\mathrm{x}$ & $\mathrm{x}$ & & \\
\hline 17 & Halecium pusillum (M. Sars, 1857) & $\mathrm{g}$ & TA & $\mathrm{p}$ & $\mathrm{f} / \mathrm{p}$ & $\mathrm{x}$ & $\mathrm{p}$ & $\mathrm{p}$ & $\mathrm{p}$ & $\mathrm{x}$ & & $\mathrm{p}$ & $\mathrm{f}$ & $\mathrm{x}$ & $\mathrm{x}$ & $\mathrm{p}$ & & $\mathrm{x}$ & \\
\hline 18 & Hydrodendron mirabile (Hincks, 1868) & $\mathrm{g}$ & MA & & & & $\mathrm{x}$ & $\mathrm{x}$ & & & & & & $\mathrm{x}$ & & $\mathrm{x}$ & $\mathrm{x}$ & & \\
\hline 19 & Aglaophenia octodonta (Heller, 1868) & $\mathrm{g}$ & MA & & $\mathrm{x}$ & $\mathrm{f}$ & $\mathrm{f}$ & $\mathrm{f}$ & $\mathrm{x}$ & $\mathrm{x}$ & & $\mathrm{x}$ & $\mathrm{f}$ & $\mathrm{x}$ & & $\mathrm{x}$ & $\mathrm{f}$ & $\mathrm{f}$ & \\
\hline 20 & Aglaophenia pluma (L., 1758) & $\mathrm{g}$ & $\mathrm{C}$ & & & & $\mathrm{f}$ & $\mathrm{f}$ & $\mathrm{x}$ & & & & & $\mathrm{x}$ & & & $\mathrm{x}$ & $\mathrm{x}$ & \\
\hline 21 & $\begin{array}{l}\text { Aglaophenia tubiformis } \\
\text { (Marktanner-Turneretscher, 1890) }\end{array}$ & $\mathrm{g}$ & MA & & & & $\mathrm{f}$ & $\mathrm{f}$ & $\mathrm{x}$ & $\mathrm{x}$ & & & $\mathrm{x}$ & $\mathrm{x}$ & $\mathrm{x}$ & $\mathrm{x}$ & $\mathrm{x}$ & $\mathrm{x}$ & \\
\hline 22 & Aglaophenia indet. & $\mathrm{g}$ & & $\mathrm{x}$ & $\mathrm{f}$ & $\mathrm{x}$ & $\mathrm{x}$ & $\mathrm{f}$ & $\mathrm{x}$ & $\mathrm{x}$ & & $\mathrm{x}$ & $\mathrm{x}$ & & $\mathrm{x}$ & $\mathrm{x}$ & $\mathrm{x}$ & $\mathrm{x}$ & \\
\hline $\begin{array}{l}23 \\
24\end{array}$ & $\begin{array}{l}K i \\
V e\end{array}$ & $\begin{array}{l}\mathrm{g} \\
\mathrm{g}\end{array}$ & $\mathrm{C}$ & $\mathrm{x}$ & $\mathrm{x}$ & $\mathrm{x}$ & $\mathrm{x}$ & $\mathrm{x}$ & $\mathrm{x}$ & $\mathrm{x}$ & & $\mathrm{x}$ & $\mathrm{x}$ & $\mathrm{x}$ & $\mathrm{x}$ & $\mathrm{x}$ & $\mathrm{x}$ & & \\
\hline 25 & aria setacea $(\mathrm{L} ., 1758)$ & $\begin{array}{l}\mathrm{g} \\
\mathrm{g}\end{array}$ & C & $\mathrm{x}$ & & & $\mathrm{x}$ & $\mathrm{x}$ & $\mathrm{x}$ & $\mathrm{x}$ & & $\mathrm{x}$ & & & & $\hat{x}$ & & $\mathrm{x}$ & \\
\hline 26 & zena disticha (Bosc, 1802) & $\mathrm{g}$ & $\mathrm{C}$ & & & & & & $\mathrm{x}$ & & & & $\mathrm{f}$ & & & & & $\mathrm{x}$ & \\
\hline 27 & Sertularella gaudichaudi (Lamouroux, 1824) & ) $\mathrm{g}$ & TA & & $\mathrm{x}$ & & $\mathrm{x}$ & $\mathrm{x}$ & $\mathrm{x}$ & $\mathrm{x}$ & & & $\mathrm{f}$ & $\mathrm{x}$ & $\mathrm{x}$ & $\mathrm{x}$ & $\mathrm{x}$ & $\mathrm{x}$ & \\
\hline 28 & Clytia noliformis (McCrady, 1859) & $\mathrm{m}$ & TA & & & $\mathrm{x}$ & $\mathrm{x}$ & & & & & & & & & & & & \\
\hline 29 & Clytia $\mathrm{s}$ & $\mathrm{m}$ & & $\mathrm{f}$ & & $\mathrm{x}$ & $\mathrm{f}$ & $\mathrm{f}$ & $\mathrm{f}$ & $\mathrm{x}$ & & $\mathrm{f}$ & & $\mathrm{x}$ & $\mathrm{x}$ & $\mathrm{f}$ & $\mathrm{f}$ & $\mathrm{f}$ & \\
\hline 30 & Obelia dichotoma (L., 1758) & $\mathrm{m}$ & $\mathrm{C}$ & & & & & $\mathrm{f}$ & $\mathrm{f}$ & & & & & & & $\mathrm{x}$ & $\mathrm{f}$ & $\mathrm{x}$ & \\
\hline 31 & Orthopyxis crenata (Hartlaub, 1901) & $\mathrm{mg}$ & $\mathrm{CT}$ & & & & & & & $\mathrm{x}$ & & & & & & & & & \\
\hline 32 & Orthopyxis integra (Macgillivray, 1842) & $\mathrm{mg}$ & $\mathrm{C}$ & $\mathrm{X}$ & $\mathrm{f}$ & $\mathrm{f}$ & $\mathrm{X}$ & & x & $\mathrm{x}$ & & $\mathrm{x}$ & $\mathrm{x}$ & $\mathrm{x}$ & $\mathrm{x}$ & $\mathrm{x}$ & $\mathrm{x}$ & & \\
\hline
\end{tabular}

TABLE 2. - Abundance and distribution of hydroid colonies on the stems of Cystoseira.

\begin{tabular}{|c|c|c|c|c|c|c|c|c|c|c|c|c|c|c|c|c|c|c|c|c|c|}
\hline \multirow{4}{*}{$\begin{array}{l}\text { site } \\
\text { category }\end{array}$} & \multirow{4}{*}{\multicolumn{2}{|c|}{$\underset{2}{\exp .}$}} & \multirow{2}{*}{\multicolumn{3}{|c|}{ C. compressa }} & \multicolumn{15}{|c|}{ a) abundance on stem/colony size (\% per category*) } & \multirow[b]{4}{*}{3} \\
\hline & & & & & & & \multirow{2}{*}{\multicolumn{3}{|c|}{$\begin{array}{l}\text { C. barb } \\
\text { exp. }\end{array}$}} & \multirow{2}{*}{\multicolumn{3}{|c|}{ shelt. }} & \multirow{2}{*}{\multicolumn{3}{|c|}{ C. amentacea }} & \multicolumn{5}{|c|}{ Cystoseira tot } & \\
\hline & & & & & shelt. & & & & & & & & & & & & exp. & & & shelt. & \\
\hline & & & 3 & 1 & 2 & 3 & 1 & 2 & 3 & 1 & 2 & 3 & 1 & 2 & 3 & 1 & 2 & 3 & 1 & 2 & \\
\hline athecata & 56 & 44 & 0 & 87 & 13 & 0 & 59 & 18 & 23 & 61 & 32 & 7 & 54 & 46 & 0 & 58 & 27 & 15 & 64 & 30 & 6 \\
\hline thecata & 46 & 37 & 17 & 74 & 22 & 4 & 32 & 39 & 29 & 57 & 35 & 8 & 37 & 42 & 21 & 38 & 39 & 23 & 62 & 31 & 7 \\
\hline total \% & 47 & 38 & 16 & 76 & 20 & 4 & 36 & 36 & 28 & 59 & 34 & 8 & 38 & 42 & 20 & 40 & 38 & 22 & 62 & 31 & 7 \\
\hline
\end{tabular}

b) distribution on stem (\% per category**)

\begin{tabular}{|c|c|c|c|c|c|c|c|c|c|c|c|c|c|c|c|c|c|c|c|c|c|}
\hline \multirow{3}{*}{$\begin{array}{l}\text { site } \\
\text { category }\end{array}$} & \multicolumn{6}{|c|}{ C. compressa } & \multicolumn{5}{|c|}{ C. barbata } & \multicolumn{4}{|c|}{ C. amentacea } & \multicolumn{5}{|c|}{ Cystoseira tot } & \multirow[b]{3}{*}{3} \\
\hline & & exp. & & & shelt. & & & exp. & & & shelt. & & & exp. & & & exp. & & & shelt. & \\
\hline & 1 & 2 & 3 & 1 & 2 & 3 & 1 & 2 & 3 & 1 & 2 & 3 & 1 & 2 & 3 & 1 & 2 & 3 & 1 & 2 & \\
\hline athecata & 80 & 20 & 0 & 69 & 19 & 12 & 70 & 27 & 3 & 37 & 49 & 14 & 70 & 15 & 15 & 73 & 23 & 4 & 40 & 46 & 14 \\
\hline thecat & 34 & 50 & 16 & 15 & 42 & 43 & 39 & 56 & 5 & 17 & 38 & 45 & 16 & 70 & 14 & 31 & 58 & 11 & 16 & 39 & 44 \\
\hline total \% & 37 & 48 & 15 & 22 & 39 & 39 & 42 & 53 & 5 & 23 & 42 & 35 & 19 & 67 & 14 & 35 & 55 & 10 & 23 & 41 & 36 \\
\hline
\end{tabular}

*1 = present, 2 = abundant, 3 = very abundant

** 1 = stipe and holdfast, $2=$ inner or intermediate part of the stem, $3=$ outer part of the stem 
a)
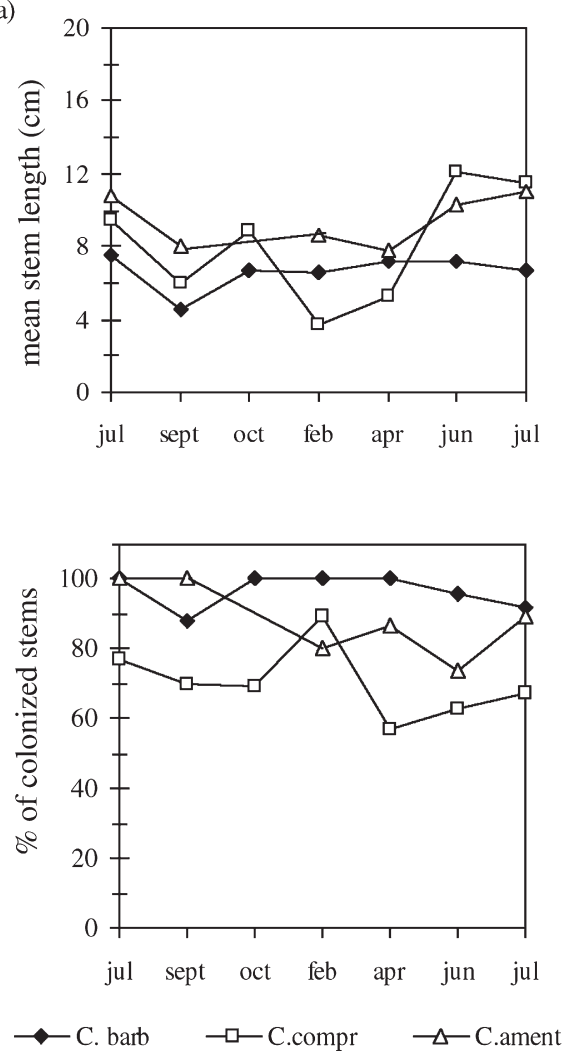

b)
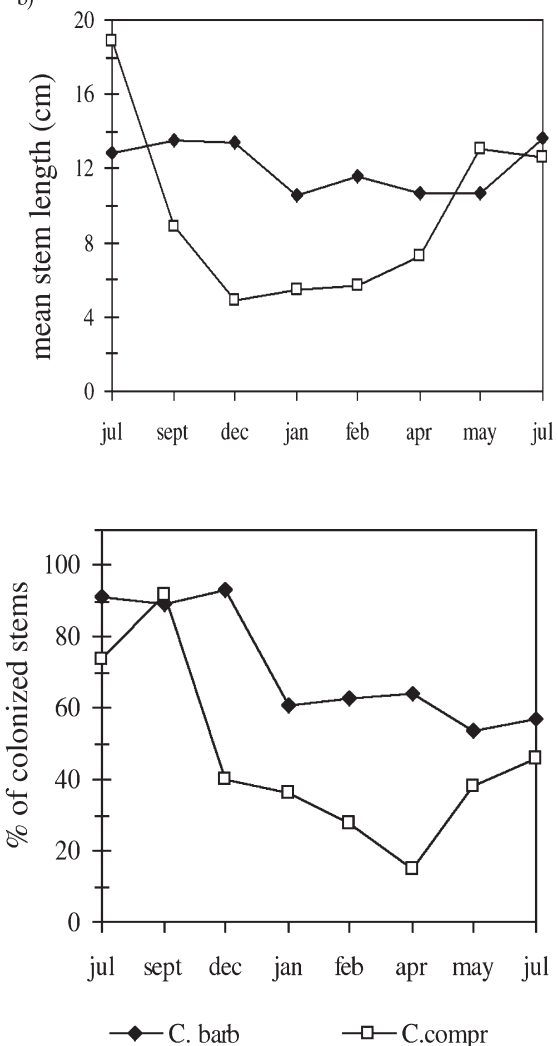

FIG. 1. - Mean algal stem length and percentage of colonised stems of Cystoseira between July 1996 and July 1997 for a) sheltered and b ) exposed site.

Decrease in colony height on Cystoseira could be observed in the arborescent species Halecium pusillum, Ventromma halecioides, Aglaophenia spp and Sertularella gaudichaudi. Colonies of the genus Aglaophenia had a much higher frequency at the wave-exposed than at the sheltered site.

\section{Hydroid diversity and dominance}

Of the 32 species of hydroids (Table 1) sixteen were found at both sites and on all three species of Cystoseira. With 27 species, the hydroids on stems of $C$. barbata from the sheltered site showed the highest species richness (Table 3). No host-specificity was found amongst the hydroids on Cystoseira.
The Shannon's diversity index (H') and Pielou's evenness index (J) for the hydroid communities are shown in Table 2. Differences in $H^{\prime}$ and $J$ on the three algal species at the two sites were not significant (randomisation test: p-value between 0.074 and 0.709).

Simpson's index of dominance $\left(\mathrm{I}_{\mathrm{dom}}\right)$ is shown in Table 4. The five dominant $\left(\mathrm{I}_{\mathrm{dom}}>1.0\right)$ species were Halecium pusillum, Aglaophenia tubiformis, Ventromma halecioides, Clytia sp and Orthopyxis integra, all of which were thecate. They comprised 34$73 \%$ of the hydroid colonies found. The subdominant species with an index of dominance between 1.0 and 0.6 were Eudendrium motzkossowskae, Hydractinia fucicola, Coryne muscoides, Cladoco-

TABLE 3. - Percentage of algal stems colonized by at least one hydroid, total number of hydroid species recorded, Shannon-Wiener diversity index and Pielou's evenness index for the hydroid communities on the three species of Cystoseira at the two sites of different wave exposure.

\begin{tabular}{lccccc}
\hline $\begin{array}{l}\text { algal substrate } \\
\text { site (exposed/sheltered) }\end{array}$ & \multicolumn{2}{c}{$\begin{array}{c}\text { C. compressa } \\
\text { shelt }\end{array}$} & exp & exp & \multicolumn{2}{c}{ C. barbata } \\
shelt & & C. amentacea \\
exp
\end{tabular}


TABLE 4. - Species and order of dominance of hydroids on Cystoseira. Dominance index Idom $=\mathrm{S}(\mathrm{n} / \mathrm{N}) * 10$, where: $\mathrm{n}=$ number of colonies of species ' $\mathrm{x}$ ', $\mathrm{N}=$ total colonies of all species in sample. Order $=$ order of dominance.

\begin{tabular}{|c|c|c|c|c|c|c|c|c|c|c|c|}
\hline \multirow[b]{3}{*}{ No. } & \multirow[b]{3}{*}{ species } & \multicolumn{4}{|c|}{ Cystoseira compressa } & \multirow{2}{*}{\multicolumn{4}{|c|}{ Cystoseira barbata }} & \multirow{2}{*}{\multicolumn{2}{|c|}{$\begin{array}{l}\text { C. amentacea } \\
\text { exposed }\end{array}$}} \\
\hline & & & osed & & ered & & & & & & \\
\hline & & order & $\mathrm{I}(\mathrm{dom})$ & order & $\mathrm{I}(\mathrm{dom})$ & order & $\mathrm{I}(\mathrm{dom})$ & order & $\mathrm{I}(\mathrm{dom})$ & order & $\mathrm{I}(\mathrm{dom})$ \\
\hline & ATHECATA & & & & & & & & & & \\
\hline 1 & Clava multicornis & - & - & 11.5 & 0.28 & & $<0.01$ & 13 & 0.1 & 16 & 0.01 \\
\hline 2 & Eudendrium glomeratum & - & - & - & - & - & - & 22 & 0.01 & - & - \\
\hline 3 & Eudendrium motzkossowskae & 10 & 0.1 & 13 & 0.16 & 15 & 0.06 & 7 & 0.75 & - & - \\
\hline 4 & Eudendrium ramosum & - & - & - & - & - & - & 16 & 0.03 & - & - \\
\hline 5 & Hydractinia fucicola & 8 & 0.4 & 7 & 0.71 & 10 & 0.34 & 10 & 0.23 & 4 & 0.96 \\
\hline 6 & Cladonema radiatum & - & - & - & - & - & - & & $<0.01$ & - & - \\
\hline 7 & Coryne muscoides & 15 & 0.03 & 16 & 0.07 & 12 & 0.21 & 6 & 0.8 & 11 & 0.11 \\
\hline 8 & Coryne pusilla & - & - & - & - & - & - & & $<0.01$ & - & - \\
\hline 9 & Sarsia producta & & $<0.01$ & - & - & - & - & - & - & - & - \\
\hline 10 & Ectopleura wrighti & - & - & - & - & - & - & & $<0.01$ & - & - \\
\hline 11 & Cladocoryne floccosa & - & - & 8 & 0.63 & 14 & 0.08 & 11 & 0.2 & - & - \\
\hline 12 & $\begin{array}{l}\text { Zanclea sp. } \\
\text { THECATA }\end{array}$ & - & - & 11.5 & 0.28 & - & - & 14 & 0.09 & 16 & 0.01 \\
\hline 13 & Mitrocoma annae & - & - & - & - & - & - & & $<0.01$ & - & - \\
\hline 14 & Eirene viridula & - & - & - & - & & & - & - & - & - \\
\hline 15 & Anthohebella parasitica & 11.5 & 0.04 & - & - & 16 & 0.04 & & $<0.01$ & 16 & 0.01 \\
\hline 16 & Halecium pusillum & 2 & 3 & 3 & 2.83 & 1 & 4.53 & 5 & 1.34 & 1 & 5.17 \\
\hline 17 & Halecium nanum & 16 & 0.02 & 10 & 0.29 & & $<0.01$ & 21 & 0.01 & 12 & 0.07 \\
\hline 18 & Hydrodendron mirabile & - & - & - & - & & $<0.01$ & 17 & 0.03 & 0 & $<0.01$ \\
\hline 19 & Aglaophenia octodonta & 7 & 0.41 & 6 & 0.8 & 4 & 0.91 & 8 & 0.47 & 9 & 0.18 \\
\hline 20 & Aglaophenia pluma & 13 & 0.04 & - & - & 13 & 0.14 & 20 & 0.02 & 14 & 0.01 \\
\hline 21 & Aglaophenia tubiformis & 1 & 4.01 & 2 & 6.92 & 3 & 1.38 & 1 & 3.61 & 3 & 1.04 \\
\hline 22 & Aglaophenia indet. & 3 & 2.69 & 5 & 1.16 & 2 & 1.96 & 3 & 1.9 & 2 & 2.35 \\
\hline 23 & Kirchenpaueria sp. & - & - & - & - & - & - & & $<0.01$ & & $<0.01$ \\
\hline 24 & Ventromma halecioides & 6 & 0.41 & 4 & 2.75 & 6 & 0.49 & 4 & 1.45 & 10 & 0.15 \\
\hline 25 & Plumularia setacea & 9 & 0.15 & 15 & 0.1 & 9 & 0.41 & 18.5 & 0.02 & 8 & 0.27 \\
\hline 26 & Dynamena disticha & - & - & - & - & 8 & 0.42 & 18.5 & 0.02 & & $<0.01$ \\
\hline 27 & Sertularella gaudichaudi & 17 & 0.02 & - & - & 5 & 0.64 & 12 & 0.11 & 6 & 0.42 \\
\hline 28 & Clytia noliformis & 14 & 0.03 & - & - & & $<0.01$ & - & - & - & - \\
\hline 29 & Clytia sp. & 5 & 0.98 & 1 & 8.02 & 7 & 0.48 & 2 & 2.5 & 5 & 0.44 \\
\hline 30 & Obelia dichotoma & 11.5 & 0.04 & 14 & 0.12 & & $<0.01$ & 15 & 0.07 & 13 & 0.03 \\
\hline 31 & Orthopyxis crenata & - & - & - & - & - & - & - & - & & $<0.01$ \\
\hline 32 & Orthopyxis integra & 4 & 1.5 & 9 & 0.33 & 11 & 0.32 & 9 & 0.43 & 7 & 0.39 \\
\hline
\end{tabular}

ryne floccosa, Aglaophenia octodonta and Sertularella gaudichaudi, which together with the dominant species comprised $55-84 \%$ of the hydroid communities.

\section{Canonical Correspondence Analysis}

The CCA ordinations were all statistically significant $(\mathrm{P}=0.01$ or $\mathrm{P}<0.001)$, as assessed by unrestricted Monte Carlo permutation tests (overall test for the first four axes for 1000 permutations). The species-environmental correlations for the first two axes of ordination ranged from 0.72 to 0.89 and the eigenvalues for the first two axes from 0.12 to 0.2 . Figure 2 summarises across both algal and hydroid species. The sites of different wave-exposure are separated by the first CCA axis in all seasons. The samples of the exposed site lie below the first axis of the plot, where colonisation is high. Most variation at the wave-exposed site is due to stem length and number of stems. Samples suggest a seasonal cycle. Samples with the lowest colonisation levels and

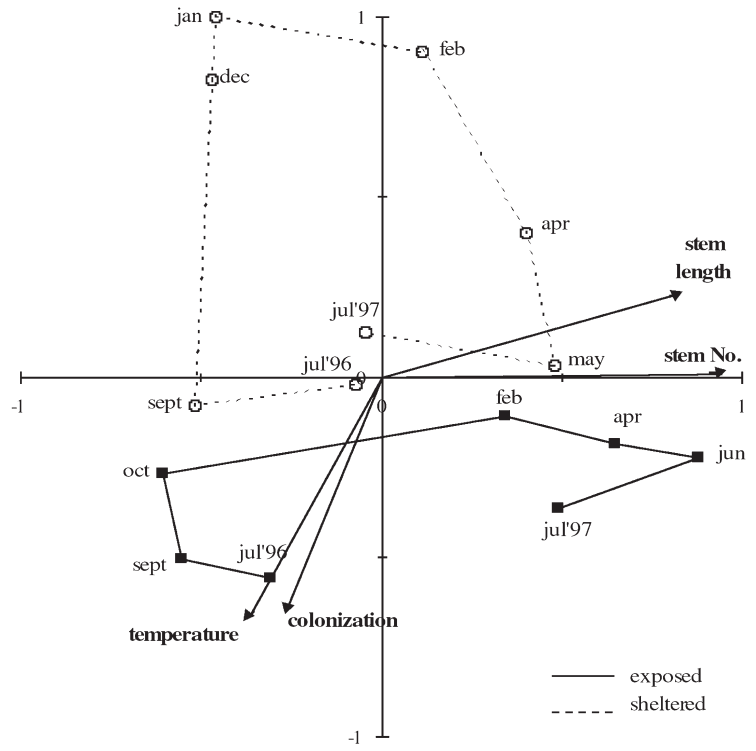

FIG. 2. - Ordination of faunal samples for exposed and sheltered sites using Analysis of Canonical Correspondence with environmental variables. $x$ and $y$ are the first two CCA axes. Arrows show direction and rates of variation among month in water temperature, $\%$ of colonised algal stems, algal stem length and number of sampled stems $(\mathrm{P}=0.01)$. 


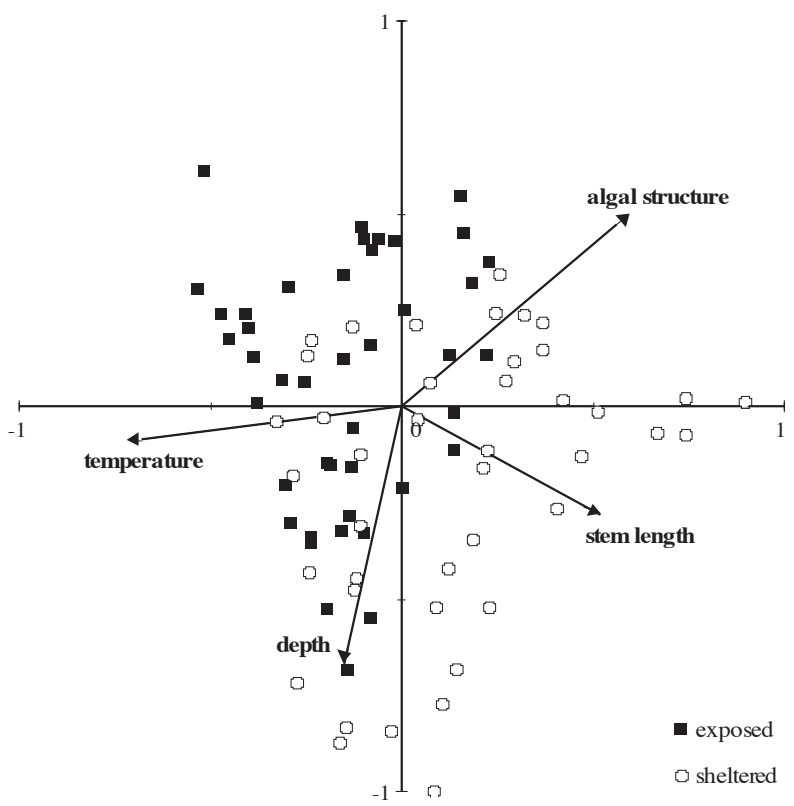

FIG. 3. - Ordination of faunal samples for exposed and sheltered sites using Analysis of Canonical Correspondence with environmental variables. $x$ and $y$ are the first two CCA axes. Arrows show direction and rates of variation among sites in water temperature, water depth, algal stem length and degree of algal structure $(\mathrm{P}=0.001)$.

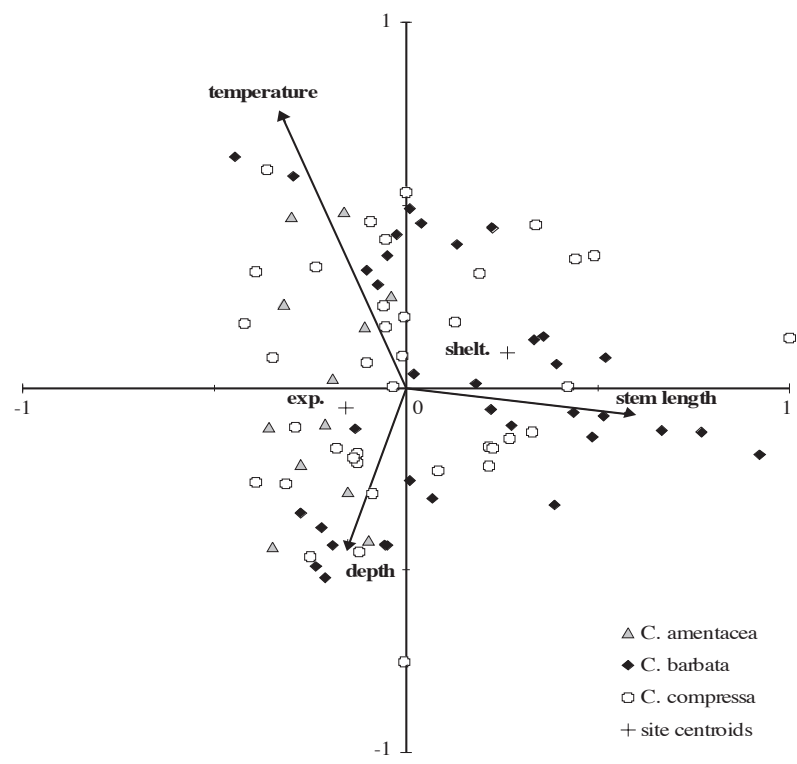

FIG. 4. - Ordination of faunal samples for Cystoseira amentacea, $C$. barbata and C. compressa using Analysis of Canonical Correspondence with environmental variables. $\mathrm{x}$ and $\mathrm{y}$ are the first two CCA axes. Arrows show direction and rates of variation among samples in water temperature, water depth and algal stem length. The sheltered and exposed sites are shown by their centroids $(\mathrm{P}=0.001)$.

temperature are the ones at the sheltered site during the cold season.

Figure 3, in which each point is the species composition at a particular site indicates that there is a tendency for samples at the sheltered site to lie

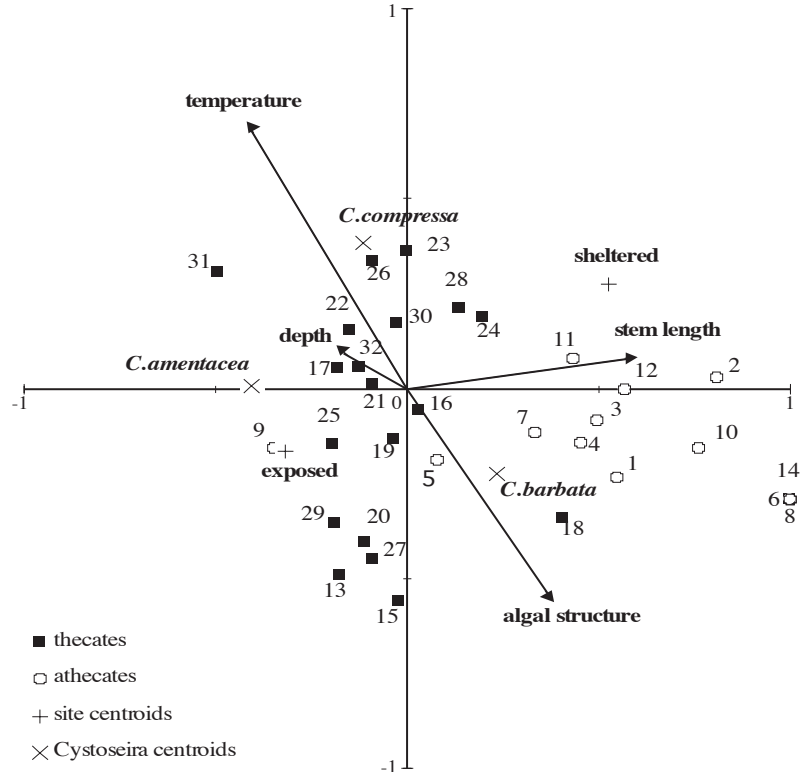

FIG. 5. - Ordination of hydroid species for athecates and thecates using Analysis of Canonical Correspondence with environmental variables. $\mathrm{x}$ and $\mathrm{y}$ are the first two CCA axes. Arrows show direction and rates of variation among samples in water temperature, water depth, algal stem length and degree of algal structure. The sheltered and exposed sites, and the three species of Cystoseira are shown by their centroids. Numbers indicate the hydroid species $(\mathrm{P}=0.001)$.

towards the lower right of the plot. This region also represents sites where algae had long stems with high structure and where the water was colder and deeper.

Figure 4, which displays differences among the three algal species, shows that samples from $C$. amentacea, which was only found at the exposed site, lie to the left of the second CCA axis, where the centroid of the exposed site is represented. Samples for $C$. barbata show a tendency to lie along the positive region of the first CCA axis, representing sites with high stem length and shelter. Variation between the samples are influenced by water temperature, stem length and depth.

The species points in Figure 5 summarise the preferences of species for different types of sites. The athecate hydroids are separated from the thecate hydroids and lie on the plot where stem length, algal structure and shelterness is high.

\section{DISCUSSION}

This study examined the seasonal and spatial distribution of epiphytic hydroids on three species of the brown algae belonging to the genus Cystoseira. Of the 32 epiphytic species of hydroids (Table 1 ) the 
most abundant species are all widespread (Boero and Bouillon, 1993). The two species endemic to the Mediterranean Sea were only found occasionally. The dominant hydroid species present on Cystoseira are well known from habitats with a high level of stress factors (Boero, 1981b; Boero and Fresi, 1986). There are no differences in diversity and evenness among the communities of the two sites and on the three species of Cystoseira, but species compositions differ.

No host-specificity was found, although host-specific hydroid species are well known from other seaweeds and seagrasses (Boero, 1981a; Hughes et al., 1991; Watson, 1992). All hydroid species epiphytic on Cystoseira, except perhaps a new species of Clytia, are well known from other biotic or hard bottom substrates. Twenty-three of the hydroid species found $(74 \%)$ are well known from other epiphytic systems in the Mediterranean Sea (Boero 1981a; Llobet et al., 1986). Substrate availability, i.e. abundance of Cystoseira, does not seem to strongly influence the epiphytic hydroid community. Algal substrate is generally more abundant at the sheltered site, but colonisation and number of species is higher, and colony size bigger at the wave-exposed site. Only on $C$. compressa does colonisation correlate with stem length. The high variance of stem length of C. compressa from the sheltered site, and therefore the high instability of substrate could have led to its low colonisation. However, the lowest monthly mean in algal stem length of $C$. compressa at the sheltered site is not lower than any mean in algal stem length at the wave-exposed site. Assuming therefore that maximal stem length is a good measure of the available space on the stems of Cystoseira, in the present study the amount of available substrate does not seem to be a limiting factor for the hydroid community.

This study provides evidence that hydroid communities on Cystoseira have a seasonal cycle. This seasonality could be dependent on substrate availability, but in this study, this factor did not seem to influence the hydroid community. As the abundance of the hydroids on Cystoseira coincides with those of the hydroids in the Mediterranean Sea in the cold season in general, their seasonality does not seem to be influenced by their special habitat. The occurrence of the most fertile colonies, the highest species richness and the period of highest hydroid abundance in the cold season, also supports this.

Hydroid abundance differed between the two sites experiencing different levels of wave-expo- sure. The stems of Cystoseira differ between sites in their length and colonisation by hydroids. At the wave-exposed site the stems of Cystoseira were probably shorter because of the higher mechanical stress. The higher levels of water movement could result in a higher frequency of stem breakage and so in a higher turnover rate, or in a limitation of growth to a certain length. Water movement supplies the organisms with food, nutrients and dissolved gases, prevents accumulation of sediments, and disperses waste products and larvae (Riedl, 1969). Therefore the stems were probably more frequently colonised, had a higher species frequency, a higher number of species per stem, and larger colonies. However, the tendency for hydroid colonies, especially of the fragile athecate species, to be distributed more towards the protected parts of the stems at the exposed site is likely to be a response to higher mechanical stress.

Another characteristic of epiphytic species is the reduced size or colony height, especially of arborescent species. This was observed among the hydroid species growing on Cystoseira. The change in morphology is also likely to be a structural response to water movement. With increasing intensity of water movement colony height decreases and population density and number of hydranths per colony increases. Because growth forms with greater hydranth density require a higher influx of nutrient particles, they tend to occupy more wave-exposed habitats (Riedl, 1971). This can be seen by examining the distribution and fertility of species with different hydranth densities. Colonies of the genus Aglaophenia, which have the highest hydranth density per colony, have a higher frequency and abundance at the exposed site than at the sheltered site. Species of the family Campanulariidae have the lowest hydranth density per colony. Among them, Clytia $\mathrm{sp}$, the most frequent species colonising Cystoseira was clearly more abundant at the sheltered site. This is underlined by the distribution of fertile colonies among the hydroids: Aglaophenia spp contribute $44 \%$ to the fertile colonies at the wave-exposed site, while campanulariid species contribute only $28 \%$. At the sheltered site the campanulariids contribute $66 \%$ and the Aglaophenia species only $11 \%$.

The hydroid communities on the three species of Cystoseira do not differ greatly. There is, however, a trend towards decreasing colonisation, hydroid abundance and colony size from $C$. barbata to $C$. amentacea to $C$. compressa. These differences could be due to host preferences by larvae which select on 
the basis of algal architecture and surface, its age or chemical substances (Nishihira, 1966; Katô et al., 1975; Amico, 1995). The influence of chemical exudates seems only to be important on the same stem where the antibacterial effect prevents colonisation of young branches. This was observed mainly on $C$. amentacea, which has more complex metabolites with a much higher antibacterial action (Amico, 1995) than C. barbata and C. compressa. The most probable factor responsible for the differences in colonisation among the three species of Cystoseira is their morphological nature. C. barbata and $C$. amentacea, which are both highly branched with many micro-habitats facilitating hydroid settlement and persistence, are also more heavily colonised than $C$. compressa, which shows a simpler morphology with its flattened and smoother branches. It can be concluded, therefore, that the highest abundance of hydroids on C. barbata is mainly due to its highly structured morphology.

Thirty-nine percent of the epiphytic hydroid community on Cystoseira comprises athecate species. This is greater than that found on Posidonia oceanica (Boero, 1981a) or Halimeda tuna (Llobet et al., 1991), where athecates comprise $23 \%$ and $14 \%$ of the hydroid community, respectively. In the Mediterranean Sea athecates generally comprise $48 \%$ of all hydroids (Boero and Bouillon, 1993). One reason why athecates are less common in epiphytic communities is the higher level of mechanical forces acting on them. Athecates are presumably more sensitive to these forces because they have no rigid thecae for support or for the polyp to retract into. This may also be the reason why they are more distributed on the more protected lower and inner parts of the stem. The higher frequency of athecate species on Cystoseira compared to other seaweeds could be due to its dendroid growth form, which offers more protected places for fragile species to withstand high mechanical stress. The dominance of thecate species among epiphytes underlines the idea that habitats with high mechanical stress are less favourable to the more fragile athecate species.

\section{CONCLUSIONS}

The hydroid species colonising Cystoseira may avoid the higher levels of competition found on other substrates, but only those species with features enabling them to persist in an environment of high mechanical stress survive as epiphytes. This is prob- ably why the dominant epiphytic hydroids on stems of the brown algae Cystoseira from the sites of this study are thecates. The factors with the greatest influence on hydroid communities occupying sites exposed to wave action rather than more sheltered sites appear to be mostly abiotic: sedimentation rate, nutrient levels, temperature and, especially water movement. Biological factors such as the structure and surface of the host and competition seem to be secondary. The importance of water movement, which in this study seemed to have the greatest influence on the hydroid community, could be due to the shallow water depth $(0.5-3 \mathrm{~m})$ and high exposure to wave action of the study site.

\section{ACKNOWLEDGEMENTS}

This project was fulfilled as part of a Masters Project of A. Faucci at the University of Basel, Switzerland under supervision of Prof. S.C. Stearns. Thanks to the people of the Zoology Laboratory at the University of Lecce and the Istituto Talassografico di Taranto, Italy for their practical advises and helps with species identification. Thanks especially to Prof. S.C. Stearns for valuable comments on the manuscript, Dita Vizoso for helps with statistics, and all the people of the Zoology Institute Basel for their useful discussions. Financial support from: C.N.R. (PRISMA 2); Amministrazione Provinciale di Lecce; M.U.R.S.T. (60\% and COFIN projects).

\section{REFERENCES}

Amico, V. - 1995. Marine brown algae of Family Cystoseiraceae: chemistry and chemotaxonomy. Phytochemistry, 39(6): 12571279.

Amico, V., G. Giaccone, P. Colombo, P. Colonna, A.M. Mannino and R. Randazzo. - 1985. Un nuovo approccio allo studio della sistematica del genere Cystoseira C.Agardh (Phaeophyta, Fucales). Boll. Acc. Gioenia Sci. Nat., 18 (326): 887-986.

Arrontes, J. - 1990. Composition, distribution on host, and seasonality of epiphytes on three intertidal algae. Bot. Mar., 33: 205211 .

Boero, F. - 1981a. Systematics and ecology of the hydroid population of two Posidonia oceanica meadows. P.S.Z.N.I: Mar. Ecol., 2(3): 181-197.

Boero F. - 1981b. Osservazioni ecologiche sugli idroidi della fascia a mitili della Riviera Ligure di Levante (I). Cah. Biol. Mar., 22: 107-117.

Boero, F. - 1984. The ecology of marine hydroids and effects of environmental factors: a review. P.S.Z.N.I: Mar. Ecol., 5(2): 93-118.

Boero, F. - 1987. Evolutionary implications of habitat selection in the hydroids of Posidonia oceanica meadows. In: J. Bouillon, F. Boero, F. Cicogna and Paul F. S. Cornelius (eds.), Modern trends in the systematics, ecology, and evolution of Hydroids and Hydromedusae, pp. 251-256. Clarendon Press, Oxford.

Boero, F. and J. Bouillon. - 1993. Zoogeography and life cycle patterns of Mediterranean hydromedusae (Cnidaria). Biol. J. Linn. 
Soc., 48: 239-266.

Boero, F and E. Fresi. - 1986. Zonation and evolution of a rocky bottom hydroid community. P.S.Z.N.I: Mar. Ecol., 7(2): 123-150.

Coma, R., I. Llobet, M. Zabala, J.-M. Gili and R.G. Hughes. - 1992. The population dynamics of Halecium petrosum and Halecium pusillum (Hydrozoa, Cnidaria), epiphytes of Halimeda tuna in the northwestern Mediterranean. In: Aspects of Hydrozoan Biology. Sci. Mar., 56(2-3): 161-169.

Cormaci, M. - 1995. Struttura e periodismo dei popolamenti a Cystoseira (Fucophyceae, Fucales) del Mediterraneo. Giorn. Bot. Ital., 129: 357-366.

Garcia Rubies, A. - 1987. Distribution of epiphytic hydroids on Posidonia sea grass. In: J. Bouillon, F. Boero, F. Cicogna and Paul F. S. Cornelius (eds.). Modern trends in the systematics, ecology, and evolution of Hydroids and Hydromedusae, pp. 143-155. Clarendon Press, Oxford.

Gili, J.-M. and R.G. Hughes. - 1995. The ecology of marine benthic Hydroids. Oceanogr. Mar. Biol. Ann. Rev. 33: 351-426.

Hayward, P.J. - 1980. Invertebrate epiphytes of coastal marine algae. In: J.H. Price et al. (eds.). The shore environment. Vol.2 Ecosystems, 17b: 761-787. Academic Press, London.

Hughes, R.G., S. Johnson and I.D. Smith. - 1991. The growth patterns of some hydroids that are obligate epiphytes of seagrass leaves. Hydrobiologia, 216/217: 205-210.

Katô, T., A.S. Kumanireng, I. Ichinose, Y. Kitahara, Y. Kakinuma, M. Nishihira and M. Katô. - 1975. Active components of Sargassum tortile effecting the settlement of swimming larvae of Coryne uchidai. Experientia, 31 (4): 433-434.

Krebs, C.J. - 1989. Ecological Methodology. Harper Collins Publishers, New York.

Llobet, I., J.-M. Gili and R.G. Hughes. - 1991. Horizontal, vertical and seasonal distributions of epiphytic Hydrozoa on the alga Halimeda tuna in the northwestern Mediterranean Sea. Mar. Biol., 110: 151-159.

Nishihira, M. - 1966. Ecological distribution of epiphytic Hydrozoa on the Tsuchiya coast near the marine biological station of Asamushi. Bull. Mar. Biol. Stat. Asamushi, 12 (4): 179-205.

Otero-Schmitt, J. and J.L. Pérez-Cirera. - 1996. Epiphytism on Cytoseira (Fucales, Phaeophyta) from the Atlantic Coast of
Northwest Spain. Bot. Mar., 39: 445-465.

Riedl, R. - 1966. Biologie der Meereshölen. Paul Parey, Hamburg und Berlin.

Riedl, R. - 1969. Marinbiologische Aspekte der Wasserbewegung. Mar. Biol. 4: 62-78.

Riedl, R. - 1971. Water movement. In: O. Kinne (ed.), Marine Ecology, pp. 1123-1156. John Wiley, New York

Russo, A.R. - 1997. Epifauna living on sublittoral seaweeds around Cyprus. Hydrobiologia, 344: 169-179.

Schultze, K., K. Janke, A. Krüss and W. Weidemann. - 1990. The macrofauna and macroflora associated with Laminaria digitata and L. hyperborea at the island of Helgoland (German Bight, North Sea). Helgol. Meeresunter., 44: 39-51.

Seed, R. - 1985. Ecological patterns in the epifaunal communities of coastal macroalgae. In: P.G. Moore and R. Seed, (eds.), The ecology of rocky coasts, pp. 22-35. Hodder and Stoughton, London.

Seed, R., M.N. Elliott, P.J.S. Boaden and R.J. O’Connor. - 1981. The composition and seasonal changes amongst the epifauna associated with Fucus serratus L. in Strangford Lough, Northern Ireland. Cah. Biol. Mar., 22: 243-266.

Solow, A.R. - 1993. A simple test for change in community structure. J. Anim. Ecol., 62: 191-193.

Ter Braak, C.J.F. - 1986. Canonical Correspondence Analysis: A new eigenvector technique for multivariate direct gradient analysis. Ecology, 67(5): 1167-1179.

Ter Braak, C.J.F. - 1988. CANOCO- a FORTRAN program for canonical community ordination by (partial) (detrended) (canonical) correspondence analysis, principal components analysis and redundancy analysis (version 2.1). Technical report LWA-88-02, TNO Institute of applied computer science, Wageningen, The Netherlands.

Watson, J.E. - 1992. The hydroid community of Amphibolis seagrasses in south-eastern and south-western Australia. In: Aspects of hydrozoan biology. Sci. Mar., 56(2-3): 217-227.

Note added in proof: Clytia sp. has been identified as Clytia viridicans (Leuckart, 1856) (see Pagliara et al., this volume) 